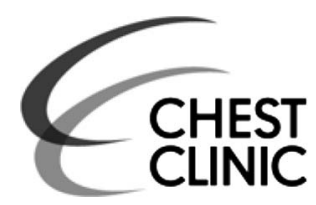
CHEST

- Additional material is published online only. To view please visit the journal online (http://dx.doi.org/10.1136/ thoraxjnl-2015-207513).

${ }^{1}$ Department of Respiratory Medicine, Nottingham University Hospitals NHS Trust, Nottingham, UK

${ }^{2}$ Division of Epidemiology and Public Health, University of Nottingham, Nottingham, UK ${ }^{3}$ Department of Respiratory Medicine, Central Manchester University Hospitals NHS Foundation Trust, Manchester UK

${ }^{4}$ British Thoracic Society, London, UK

\section{Correspondence to} Dr Priya Daniel, Department of Respiratory Medicine, Nottingham University Hospitals NHS Trust, David Evans Building, City Hospital Campus, Hucknall Road, Nottingham NG51PB, UK; priyasosha.daniel@nuh.nhs.uk

Received 1 July 2015 Revised 5 October 2015 Accepted 19 October 2015 Published Online First 11 November 2015

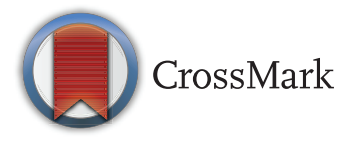

To cite: Daniel $P$, Rodrigo C, Mckeever TM, et al. Thorax 2016;71: 568-570.

\title{
Time to first antibiotic and mortality in adults hospitalised with community-acquired pneumonia: a matched-propensity analysis
}

\author{
Priya Daniel, ${ }^{1}$ Chamira Rodrigo, ${ }_{1}$ Tricia M Mckeever, ${ }^{2}$ Mark Woodhead, ${ }^{3}$ \\ Sally Welham, ${ }^{4}$ Wei Shen Lim, ${ }^{1}$ on behalf of the British Thoracic Society
}

\section{ABSTRACT}

A matched-propensity analysis of national data from the British Thoracic Society community-acquired pneumonia audit was conducted ( $n=13725)$. Overall, time to first antibiotic (TFA) was $\leq 4 \mathrm{~h}$ in $63 \%$. Adjusted 30-day inpatient (IP) mortality was lower for adults with TFA $\leq 4 \mathrm{~h}$ compared with TFA $>4 \mathrm{~h}$ (adjusted OR 0.84 , $95 \% \mathrm{Cl} 0.74$ to $0.94 ; p=0.003)$. Increasing TFA was associated with greater OR of 30-day IP mortality ( $p$ value for trend $=0.001$ ), but no TFA threshold was evident. Although we found an association between TFA and mortality, we cannot say whether this is causal or whether TFA might just be a quality measure for overall or other processes of care.

\section{INTRODUCTION}

The British Thoracic Society (BTS) communityacquired pneumonia (CAP) guideline and the National Institute for Health and Care Excellence (NICE) pneumonia guideline recommend administration of antibiotics within $4 \mathrm{~h}$ of presentation to hospital for adults with radiologically confirmed CAP. $^{12}$ However, the studies cited in support of these recommendations have been variously criticised for their restrictions on included age groups, lack of adjustment for disease severity, small sample sizes and single-centre designs. Other studies have also failed to show a mortality benefit from early administration of antibiotics in adults hospitalised with $\mathrm{CAP}^{3}{ }^{4}$

The resources required to treat patients admitted to hospital rapidly within a specific time frame are considerable. Therefore, robust evidence to support the prioritisation of patients with CAP for early treatment is desirable. However, a clinical trial to address this issue is unlikely to be conducted given inherent ethical and practical constraints. Consequently, large cohort studies in representative populations offer the best possible chance of producing meaningful results on the association between time to first antibiotics (TFA) and mortality.

\section{METHODS}

\section{Study population}

Trusts across England and Wales were invited to participate in the BTS national adult CAP audit during the period from 1 December to 31 January for years 2009/2010, 2010/2011, 2011/2012 and 2012/2013; data were submitted from 188 institutions. Immunocompetent adults aged $\geq 16$ years hospitalised with CAP (defined as new infiltrates on chest X-ray with symptoms suggestive of lower respiratory tract infection and treated by the admitting team as CAP) were included. All patients were treated at the discretion of the clinical teams. Data were entered anonymously using a secure standardised web-based system. TFA and 30-day inpatient (IP) mortality was recorded by the data collector in the participating institution. The BTS Professional and Operational Standards Committee approved the protocol; ethical approval was not required for the conduct of the audit.

TFA was defined as the interval between time of admission and time of first administration of antibiotic after admission. Adults who received their first antibiotic dose $>36 \mathrm{~h}$ after admission were excluded as they were likely to have had atypical presentations making measurement of the effects of time-sensitive processes of care inaccurate. ${ }^{5}$ Adults who died within $24 \mathrm{~h}$ of admission were excluded (in keeping with other studies) ${ }^{5}$ as TFA was not expected to modify disease outcome within such a short time.

\section{Statistical analyses}

Descriptive analyses were performed using Stata/IC V.13.1 (StataCorp, 2013). Pearson's $\chi^{2}$ test was used to compare categorical variables. The baseline characteristics, disease severity, treatment and 30-day IP mortality of adults with TFA $\leq 4$ and $>4 \mathrm{~h}$ were compared. The independent association between TFA $\leq 4 \mathrm{~h}$ and 30-day IP mortality was examined using a propensity matched model. The propensity score derivation model used covariables derived from an initial multivariable logistical regression model, where those covariables significantly associated with 30-day IP mortality and/or that led to a $>10 \%$ change in the regression coefficient were retained. The final propensity score model used the following covariables: age, variables within the CURB65 score (confusion, urea $>7 \mathrm{mmol} / \mathrm{L}$, respiratory rate $\geq 30 / \mathrm{min}$, blood pressure $<90 \mathrm{~mm} \mathrm{Hg}$ systolic or $\leq 60 \mathrm{~mm} \mathrm{Hg}$ diastolic), individual comorbidities (congestive cardiac failure (CCF), chronic heart disease excluding CCF, liver disease, chronic kidney disease (CKD), malignancy, diabetes and dementia), intravenous antibiotic use, critical care unit admission and combination $\beta$-lactam/macrolide antibiotic. Propensity scores were derived for all subjects with single nearest neighbour matching and conditional logistic 
regression used to obtain propensity-matched estimates. A secondary propensity analysis was performed to investigate the relationship between TFA (categorised as 0-4, 4-8, 8-12 and $>12 \mathrm{~h}$ ) and 30-day IP mortality.

\section{RESULTS}

Of 17403 individuals, data regarding timing of antibiotic administration were missing for 2321 adults, and mortality data were unavailable for a further 57. Of the remaining adults, 558 died within $24 \mathrm{~h}$ of admission, and 742 received their first antibiotic dose $\geq 36 \mathrm{~h}$ following admission, leaving 13725 adults. The median age of the study cohort was 75 years (IQR 60.0$85.0)$, and 6699 (48.8\%) were male.

Pneumonia severity was low (CURB65 score $\leq 1$ ), moderate (CURB65 score=2) and high (CURB65 score $\geq 3$ ) in 6045 (44.0\%), $4023(29.3 \%)$ and 3657 (26.6\%) patients, respectively. A total of $861(6.3 \%)$ individuals required admission to a critical care unit and 30-day IP mortality was $15.0 \%$. Median TFA was $3.1 \mathrm{~h}$ (IQR 1.8-5.7). The first dose of antibiotic was administered in $\leq 4,4-8,8-12$ and $>12 \mathrm{~h}$ following hospital admission in 8642 (63.0\%), 2913 (21.2\%), 976 (7.1\%) and 1194 $(8.7 \%)$ adults, respectively.

\section{Baseline characteristics and disease severity}

Adults with TFA $\leq 4 \mathrm{~h}$ were significantly younger (median age 75 years (IQR 60.0-84.0) vs 77 years (IQR 61.0-85.0); $\mathrm{p}<0.001)$, less likely to have coexisting stroke disease $(9.3 \%$ vs $10.6 \%)$, CKD (6.7\% vs $7.8 \%)$ and chronic heart disease excluding CCF $(21.3 \%$ vs $22.9 \%)$, but more likely to have COPD (23.1\% vs $20.0 \%)$ compared with adults who had TFA $>4$ h (see online supplementary table S1). Pneumonia severity was significantly higher in adults with TFA $\leq 4 \mathrm{~h}$ ( $\mathrm{p}$ value for trend for increasing CURB65 score $<0.001)$ with both intravenous antibiotic therapy $(82.0 \%$ vs $64.9 \%$; OR $2.46,95 \%$ CI 2.27 to 2.67 ; $\mathrm{p}<0.001)$ and combination $\beta$-lactam/macrolide therapy $(53.5 \%$ vs $46.8 \%$; OR $1.31,95 \%$ CI 1.22 to $1.40 ; \mathrm{p}<0.001$ ) being significantly more likely in these patients compared with those who had TFA $>4 \mathrm{~h}$. Admission to a critical care unit was required for $639(7.4 \%)$ adults with TFA $\leq 4 \mathrm{~h}$ vs $222(4.4 \%)$ adults with TFA $>4$ h (OR 1.75, 95\% CI 1.49 to 2.04; $\mathrm{p}<0.001)$.

\section{Mortality}

Propensity score adjusted 30-day IP mortality was significantly lower for adults with TFA $\leq 4 \mathrm{~h}$ compared with TFA $>4 \mathrm{~h}$

Figure 1 Propensity adjusted OR of 30-day inpatient (IP) mortality with increasing time to first antibiotic (TFA) compared with TFA $\leq 4 \mathrm{~h}$.
(OR $0.84,95 \%$ CI 0.74 to $0.94 ; \mathrm{p}=0.003$ ). Increasing delay in antibiotic administration in comparison with TFA $0-4 \mathrm{~h}$ was associated with greater OR of mortality; TFA 4-8 h: adjusted OR $1.09,95 \%$ CI 0.94 to 1.27 ; TFA $8-12$ h: adjusted OR 1.30, 95\% CI 0.99 to 1.70 and TFA >12 h: adjusted OR 1.41, 95\% CI 1.10 to 1.80 ( $\mathrm{p}$ value for trend 0.001 ) (figure 1 ).

\section{DISCUSSION}

The primary finding of this analysis is that in adults hospitalised with CAP, administration of the first antibiotic dose within $4 \mathrm{~h}$ of admission was associated with a $16 \%$ lower adjusted 30 -day IP mortality compared with later antibiotic administration. In addition, we found that increasing TFA was associated with increasing OR of mortality; no threshold for TFA was evident.

A previous retrospective analysis using the US 'Medicaid cohort' of adults aged $\geq 65$ years hospitalised with CAP $(n=13771)$ found a similar protective effect on 30-day mortality from antibiotics administered within 4 vs $>4$ h of arrival at hospital (adjusted OR 0.85, 95\% CI 0.76 to 0.95$).^{5}$ Our analysis extends these findings to adults of all ages. In contrast, a cross-sectional analysis of the US Nationwide Inpatient Sample database of $>95000$ adults with a coding diagnosis of pneumonia did not show a mortality benefit from earlier administration of antibiotics; however, the available data did not permit adjustment for pneumonia severity. ${ }^{4}$

In acute medical settings, priority of care is usually given to the sickest patients. Accordingly, we observed that patients with TFA $\leq 4 \mathrm{~h}$ had higher pneumonia severity and were more likely to receive treatments such as intravenous combination antibiotics and critical care support. Despite a matched-propensity analysis, the observed association of TFA with mortality may have been due to factors that could not be included in the propensity analysis such as clinical presentation phenotypes that made CAP diagnosis more difficult and thus delayed, and the combined effect of early clinical interventions, including factors not measured in this study such as gas exchange management strategies and fluid administration. In these respects, TFA may be serving as a quality measure for processes of care.

The strengths of this analysis are the inclusion of a large representative population of adults hospitalised with CAP and adjustment for major potential confounding variables. Data regarding preadmission antibiotics were not available; however, based on UK clinical practice, preadmission antibiotic use would not be expected to influence TFA. Instead, inclusion of adults

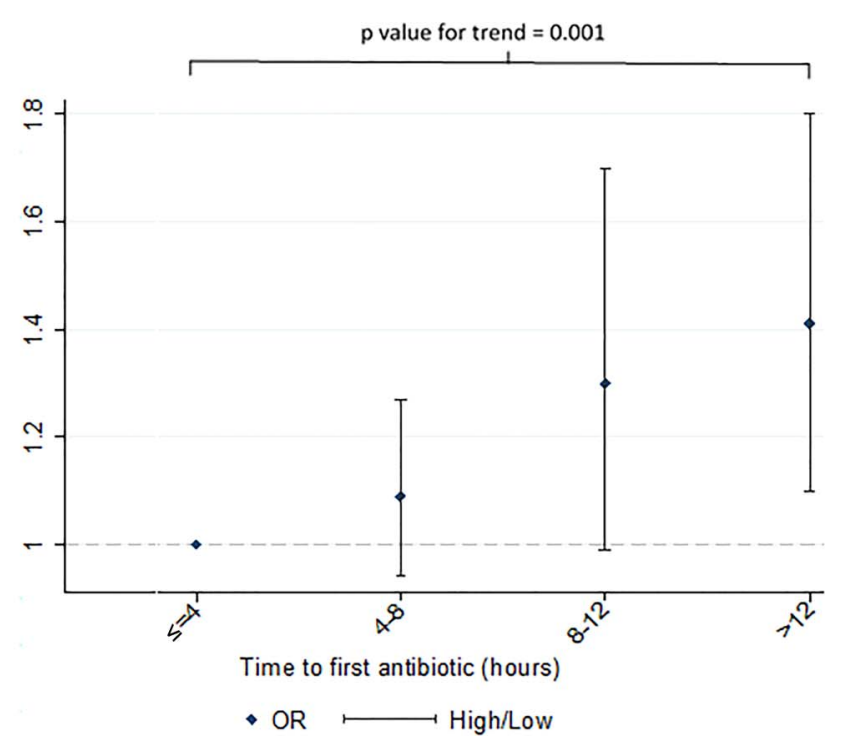




\section{Chest clinic}

who received preadmission antibiotics may have blunted the size of benefit observed. Potential weaknesses include the possibility of case selection bias in some institutions and lack of independent diagnosis confirmation; this effect is minimised by the sample size analysed.

A clinical trial to confirm the findings from this study is desirable, but would likely face considerable ethical and practical challenges.

\section{Implications of findings}

Given that nationally, $37 \%$ of patients with CAP did not receive antibiotics within $4 \mathrm{~h}$ of admission, this analysis suggests there is room for improvement. Any drive to increase the proportion receiving early antibiotics should be balanced by minimising inappropriate antibiotic use, not least through avoiding the misdiagnosis of CAP. The findings of this analysis support the 2014 NICE pneumonia guideline recommendation for health services to 'put in place processes to allow diagnosis (including X-rays) and treatment of community-acquired pneumonia within 4 hours of presentation to hospital'. They also dovetail with the Department of Health's $4 \mathrm{~h}$ target for emergency departments, and within this setting is aligned with the Infectious Diseases Society of America CAP guideline recommendation that 'first antibiotic dose should be administered while still in the emergency department'.

Acknowledgements The authors would like to thank the British Thoracic Society (BTS) audit team for their invaluable efforts in conducting the audit and Puja Myles for her statistical expertise. This paper has been prepared by the authors on behalf of the BTS, and the efforts by clinical health professionals across the UK in data collection and participation in this audit are gratefully acknowledged.

Contributors WSL and SW were instrumental in organising the British Thoracic Society national community acquired pneumonia audit; PD and CR analysed and interpreted the data, and drafted the article; TMM provided statistical input; TMM, MW, SW and WSL critically evaluated the article; all authors revised and reviewed the article prior to final approval.

Competing interests PD has received salaries funded by an unrestricted grant from Pfizer. CR has received salaries partly funded by an unrestricted grant from Pfizer and National Institute for Health Research (NIHR) grant. WSL has received funding from the NIHR for pandemic influenza research and unrestricted research funding from Pfizer for a study in pneumonia.

Provenance and peer review Not commissioned; externally peer reviewed.

\section{REFERENCES}

1 Lim WS, Baudouin SV, George RC, et al. BTS guidelines for the management of community acquired pneumonia in adults; an update 2009. Thorax 2009;64(Suppl III):iii1-55.

2 National Institute for Health and Clinical Excellence. Pneumonia: diagnosis and management of community- and hospital-acquired pneumonia in adults. London: National Clinical Guideline Centre, 2014.

3 Bruns AHW, Oosterheert JJ, Hustinx WNM, et al. Time for first antibiotic dose is not predictive for the early clinical failure of moderate-severe community-acquired pneumonia. Eur J Clin Microbiol Infect Dis 2009;28:913-9.

4 Quattromani E, Powell ES, Khare RK, et al. Hospital-reported data on the pneumonia quality measure "time to first antibiotic dose" is not associated with inpatient mortality: results of a nationwide cross-sectional analysis. Acad Emerg Med 2011;18:496-503.

5 Houck PM, Bratzler DW, Nsa W, et al. Timing of antibiotic administration and outcomes for Medicare patients hospitalized with community-acquired pneumonia. Arch Intern Med 2004;164:637-44. 